\title{
Use of information telecommunications technology in asthma subjects
}

\author{
Tilen Dolinar, Lidija Jakupović, Urška Ugovšek, Andrej Starc \\ University of Ljubljana, Faculty of Health Sciences, Zdravstvena pot 5, 1000 Ljubljana, Slovenia \\ tilen.dolinar@gmail.com, lidija.jakupovic@gmail.com, ugovseku@gmail.com, \\ andrej.starc34@gmail.com
}

\section{Abstract}

Introduction: Asthma, a chronic respiratory disease affects approximately 235-334 million people worldwide. It is one of the most common pulmonary diseases in adults. Despite extensive effective treatment that has existed for decades, most asthma patients still have uncontrolled symptoms. Health information and communication technology has been used in healthcare to persuade the self-management of various chronic diseases. Mobile health applications could provide inexpensive and clinically effective asthma control measures. Methods: We used a descriptive method of research with a review of Slovene and foreign literature. The literature search was conducted from April 2020 to May 2020. The age of the literature was limited from 2010 to 2020. The literature was searched in the CochraneLibrary, CINAHL, PubMed and MedNar databases using keywords: information and communication technology, asthma, self- management, chronicillness, application. In the search for literature with a combination of the following keywords: information and communication technology, asthma, self-management, chronic disease, application. The selection criteria was the availability of the text in its entirety and the literature published in the period from 2010 to 2020 . Articles that do not have a complete article structure, articles that do not relate to the topic of asthma were excluded. 16 articles were reviewed, 8 articles were excluded. Results: Mobile applications promise to improve behavior in asthma patients through self-management. Researchers report that about $2 / 3$ of patients have expressed interest in using an asthma management app to improve the inhaler grip, a well-known problem among asthma patients. Currently, mobile applications allow patients to monitor and manage their illness, obtain data, educate about the disease, and improve their health behavior. In addition to applications, smart inhalers could be put into use that improve the patient's quality of life and limit the 
excess use of health care. Discussion and conclusions: Information and communication technology in healthcare could be used in chronic

diseases such as asthma, as they are relatively cost effective and can have a significant impact on improving chronic disease management and human health.

\section{Keywords: information and communication technology, asthma, self- management, chronic illness, application}

\section{Introduction}

Asthma, a chronic respiratory disease, affects approximately 235-334 million people worldwide. It is one of the most common pulmonary diseases in adults. Despite the widespread of evidence-based guidelines and effective treatment that has existed for decades, most asthma patients still have uncontrolled symptoms. A patient with asthma has daily symptoms in a short-acting inhaler more than twice a week, wakes up at night due to symptoms or should definitely enter activities due to asthma. Sharing measures self-management of asthma management are key to reducing the harmful physical and economic impacts associated with this disease (Pool et al., 2017). Because asthma causes prolonged inflammation in the lungs, airway involvement severely restricts airflow during respiration, causing the patient to have difficulty breathing. Patients with asthma need two types of inhalation medications: for prevention seizures take inhaled corticosteroids to suppress the inflammation and when symptoms such as coughing or wheezing occur, all bronchodilators known as a soothing or rescue state. The most common problems that occur are improper grip of the inhaler, improper use of the inhalation itself and thus reduced absorption of the drug. The goal of asthma treatment should be dosed to maintain disease control and treatment. This requires an approach tailored to pharmacological treatment, patient education, a written action plan, training on the proper use of inhalers, and a review of inhaler technique at each outpatient visit (Zhifang et al., 2019).

International guidelines emphasize the importance of a partnership between patients and healthcare professionals. Despite all these claims, many health professionals do not pay sufficient attention to education and self-management (Zhifang et al., 2019). There is evidence that patients are not prescribed enough check-ups and do not have appropriate advice and instructions, most often this includes proper use of the inhaler. It is estimated that only $55 \%$ of adults with asthma learn to recognize early asthma symptoms, only $47 \%$ receive instructions to change the environment in which they live to improve asthma control, and only $33 \%$ have ever received an asthma treatment education plan (Pool et al., 2017).

\section{Methods}

In order to present the theoretical background and achieve the purpose of the work, a critical review of professional and scientific Slovenian and English lit- 
erature was first performed. A literature search was conducted from April 2020 to May 2020. Literature was searched in the CochraneLibrary, CINAHL, PubMed, and MedNar databases. We used literature published between 2010 and 2020. We searched for literature with various combinations of the following keywords: information and communication technology, asthma, self-management, chronic illness, application. When searching for Slovenian literature, we used combinations of the following keywords: information and communication technology, asthma, self-management, chronic disease, application. The selection criteria was the accessibility of the text as a whole. Based on the existing literature, we analyzed the use of information and telecommunication technology for people with asthma.

\section{Results}

Asthma is the most common chronic disease among children and also affects millions of adults. The U.S. health care system costs about $\$ 56$ billion a year due to its high prevalence and continued treatment throughout the lifetime of most asthma patients. Health information and communication technology has been used in healthcare to persuade the self-management of various chronic diseases such as asthma, diabetes, chronic obstructive pulmonary disease, etc. In particular, mobile health applications could provide low-cost and clinically effective asthma treatment measures. Applications could allow patients to self-manage asthma on a daily basis providing evidence-based interventions. Research has shown that procedures such as disseminating educational methods and tools to monitor symptoms improve a patient's quality of life and limit the overuse of health care services. Data obtained from mobile phone sensors and medical devices such as smart inhalers can be used to implement self-management measures tailored to the specific needs of patients (Tinschert et al., 2017).

As the use of mobile devices and smartphones becomes more common, patients can use asthma self-management apps. Currently, applications on mobile devices can enable patients to monitor and control disease, obtain data, educate about the disease, and improve health behavior (Zhifang et al., 2019). Mobile apps promise to improve behavior for asthma patients through self-management, as they can be easily incorporated into daily life. Smart mobile devices have many advantages, one of which is that they are usually always at hand, they are portable and are low cost. Researchers report that approximately $2 / 3$ of patients have expressed interest in using an asthma management app to improve inhaler adhesion (Jácome et al., 2019).

The potential of applications to improve asthma self-management varies greatly between applications. Physicians and asthmatics should therefore read application reviews carefully before deciding which application to recommend or use. In addition, currently available asthma applications unfortunately do not take full advantage of today's technology. (Tinschert et al., 2017). Traditional self-management programs should include a written action plan on 
how to identify and respond to asthma exacerbations, an individualized treatment plan, including self-monitoring and goal setting, medicine management including warnings and reminders and patient education (Honkoop, 2017).

Asthma subjects could use smart inhalers too. They are connected to a smartphone with a Bluetooth connection, remind patients when to take medicines, collect data to help with care, and improve patients commitment to taking asthma therapies. Smart inhalers contain a battery and measuring sensors which identifies the dose, and if a dose has been missed, they send patients reminders of missed doses. Sensors that measure the inhalation profile confirm that the dose was inhaled along with useful information about the inhaler technique. They also record the time of activation (Henry, 2019).

\section{Discussion}

Asthmatics could use mobile applications that allow patients to self-manage asthma on a daily basis providing evidence-based interventions. Using asthma apps as part of the set of strategies available to healthcare providers to improve quality of life among asthmatics, but it is hard to decide which app is the most suitable (Tinschert et al., 2017). Because research has shown that the inhalation technique is still a major problem for asthmatics, the use of smart inhalers that can recognize adhesion and inhalation technique is advised (Henry, 2019).

\section{Conclusions}

Given that chronic diseases account for as much as $75 \%$ of health care costs, it is crucial to identify simple tools to help patients care for them and improve outcomes. Online tools could also be used for other chronic diseases, as they are relatively cost-effective and can have a significant impact on improving the management of chronic diseases and human health. ICT can improve asthma control and the quality of life of asthmatics (Pool et al., 2017).

\section{References}

TINSCHERT, P., JAKOB, R., BARATA, F., KRAMER, J.N. and KOWATSCH,

T., 2017. The potential of mobile apps for improving asthma self-management: a review of publicly available and well-adopted asthma apps. JMIR MhealthUhealth vol. 5, no. 8. [viewed 8 March 2020]. Available from: Doi: https://doi.org/10.2196/mhealth.7177 .

GUAN, Z., SUN, L., XIAO, Q. and WANG, Y., 2019. Constructing assessment framework for the quality of asthma smart phone applications. BMC Med InformDecis Mak., vol. 19, no. 192, pp: 1-17. [viewed 8 March 2020]. Available from: doi: https://doi.org/10.1186/s12911-019-0923-8.

JÁCOME, C., AlmEIDA, R., PEREIRA, A,M,, ARAÚJO, L., CORREIA, M., PEREIRA, M., et al. 2019. Asthma apps use and interest among patients with asthma: a multicentre study. J InvestigAllergolClinImmunol, vol. 
3o, no. 2, pp: 1-8, [viewed 9 March 2020]. Available from: doi: https://doi. $\operatorname{org} / 10.18176 /$ jiaci.o456.

HONKOOP, P.J., SIMPSON, A., BONINI, M., SNOECK-STROBAND, J.B., MEAH, S., FAN CHUNG, K. et al., 2017. MyAirCoach: the use of home-monitoring and mHealth systems to predict deterioration in asthma control and the occurrence of asthma exacerbations; study protocol of anobservational study. BMJ Open, vol. 7, no. 1, pp: 1-7, [viewed 9 March 2020]. Available from: doi: https://doi.org/10.1136/bmjopen-2016-013935.

POOL, A.C., KRASCHNEWSKI, J.L., POGER, J.M., SMYTH, J., STUCKEY, H.L., CRAIG, T.J., et al. 2017. Impact of online patient reminders to improve asthma care: A randomized controlled trial. PloSOne, vol. 12, no. 2, pp: 1-17, [viewed 9 March 2020]. Available from: doi: https://doi. org/10.1371/journal.pone.0170447.

HENRY, C., AUDIBERT, R., KELLER, M., QUAGLIA, B., VECELLIO, L. and ROCHE, N., 2019. Real-lifeinhaler adherence andtechnique: Time to getsmarter! Respir Med, vol. 158, pp: 24-32, [viewed 8 March 2020]. Available from: https://doi.org/10.1016/j.rmed.2019.09.008. 